\title{
Brucella Abortus Vaccines: Use in Control Programs and Immune Response
}

\section{Dorneles EMS, Oliveria LF and Lage AP*}

Laboratório de Bacteriologia Aplicada, Departamento de Medicina Veterinária Preventiva, Escola de Veterinária, Universidade Federal de Minas Gerais, Brazil

*Corresponding author: Andrey Pereira Lage, Laboratório de Bacteriologia Aplicada, Departamento de Medicina Veterinária Preventiva, Escola de Veterinária, Universidade Federal de Minas Gerais, Brazil

Received: December 16, 2016; Accepted: February 24, 2017; Published: February 27, 2017

\begin{abstract}
Brucella spp. is a highly infectious pathogen that affects numerous livestock and wild animal species besides humans, and that although eradicated in some countries, remains one of the most economically important zoonosis worldwide. Strain 19 and RB51 are the two Brucella abortus vaccine strains more largely used in the control of brucellosis in cattle worldwide, being effective in the prevention of abortion and infection, besides offering long lasting protection. Here, we reviewed the main aspects of the $B$. abortus vaccines and their use in brucellosis control and eradication programs over the years and the current understanding in the immune response triggered by these two $B$. abortus vaccines, especially in cattle.
\end{abstract}

Keywords: S19; RB51; Vaccination; Brucellosis prevention.

\section{Introduction}

Brucellosis is a chronic zoonotic disease of great importance in public and animal health, being caused by Gram-negative, non-sporeforming, non-motile and facultative intracellular bacteria belonging to the genus Brucella [1,2]. Brucella spp. is a highly infectious pathogen that affects numerous livestock (cattle, goats, sheep, pigs, dogs, horses and cats) and wild (dolphins, whales, rodents, camels, antelopes, bison, elk) animal species, besides humans [2]. Although eradicated in some countries, it remains one of the most economically important zoonosis worldwide [2,3].

Cattle are mainly affected by Brucella abortus and the clinical signs of this infection are primarily related to reproductive problems, mainly abortion at the late pregnancy, reaching up to $80 \%$ in a susceptible herd at the time of introduction of the disease $[2,4,5]$. Besides abortion, the disease also causes in cows and their products: stillbirth, birth of weak calves, retained placenta, temporary or permanent infertility, perinatal mortality and chronic or diffuse interstitial mastitis $[4,6]$. In bulls, the disease also affects primarily the reproductive system, causing orchitis, epididymitis, decreased sperm quality and subfertility or infertility [4]. Articular disorders are also frequent in cattle and in other host animal species infected by Brucella spp. [4].

One of the most effective measures to reduce the prevalence of bovine brucellosis that has been successfully used in many control programs is the vaccination $[7,8]$. Strains 19 and RB51 are the two $B$. abortus vaccine strains more largely used in the control of brucellosis in cattle worldwide, being effective in the prevention of abortion and infection, besides offering long lasting protection [9-12]. Recently, it has been suggested that immune mechanism used by these vaccine to induce protection in cattle is based in a strong and complex immune response dominated by $\mathrm{T}$ helper type 1 (Th1) cells [13]. This response is chiefly characterized by IFN- $\gamma$ produced by $\mathrm{CD} 4^{+}$ T-cells and cytotoxicity of $\mathrm{CD} 8^{+} \mathrm{T}$-cells [13-15]. Here, we reviewed the main aspects of the $B$. abortus vaccines and their use in brucellosis control and eradication programs over the years and the current understanding of the immune response triggered by these B. abortus vaccines, particularly in cattle.

\section{B. abortus Vaccines and Vaccination}

Attenuated B. abortus strains have demonstrated the best results in the prevention of bovine brucellosis, probably because these vaccines are able to multiply within animals for a short period of time and thereby induce a strong and protective cellular immune response. Massive vaccination against brucellosis in cattle have been performed employing few vaccine strains, S19, RB51, 45/20 and SR82, although many $B$. abortus vaccine candidates have been developed over the last years, such as DNA, subunit, recombinant $B$. abortus and recombinant vector vaccines [8]. The $45 / 20$ was a bacterin used in some European countries, but the variability in reported protection, along with unpredictable serological effects and the occurrence of reactions at the site of vaccine injection in some animals led to the interruption of the use of this vaccine [16]. The SR82 strain is a live attenuated vaccine used since 1974 by the former Union of Soviet Socialist Republics (USSR) for bovine brucellosis control. Currently, the SR82 strain is still massively used in the Russian Federation, Azerbaijan, Tajikistan and other countries in the region [17]. Recombinant vaccines are part of the continuous efforts to reach a safer and more effective B. abortus vaccine, with the potential to be the future of cattle and human brucellosis control. However, many studies are still needed to achieve the ideal vaccine against brucellosis. The majority of these genetically engineered vaccines was developed and tested using mouse models and they have not been tested or were not protective in cattle [8]. Among the live modified B. abortus vaccines, S19 and RB51, are the most widely used strains around the world.

Strain 19 was the first $B$. abortus vaccine to be extensively employed for bovine brucellosis control and is still used in many countries. B. abortus S19 proved to be an efficacious vaccine under experimental tests in cattle $[11,12]$, as well as under field conditions $[18,19]$. In the experimental efficacy test of $B$. abortus vaccines in cattle after vaccination and challenge with virulent $\mathrm{B}$. abortus, protection is measured by significant reduction in the incidence of abortions 
and significant reduction in colonization of organs by challenging bacteria in vaccinated animals compared to unvaccinated animals. Protection against abortion mitigates losses to producers, whereas the lowest colonization of organs decreases the chance of transmission of the disease to other animals or to humans. The main characteristics of S19 are the stable low pathogenicity, relatively high immunogenicity and moderate antigenicity [20]. However, as a smooth attenuated B. abortus biovar 1, S19 induces antibody response that cannot be distinguished from the antibody response induced by infection with field strains. This smooth phenotype is associated with the O-side chain, an immunodominant antigen in the Lipopolysaccharide (LPS), to which the majority of antibodies resulting from S19 immunization or natural infection are directed [21]. Since residual antibody titers increase with the age at which the animal is vaccinated, vaccination of cattle with S19 is usually restricted to animals aged between 3 to 8 months, in order to mitigate the interference of anti-O-side chain antibodies in the routine diagnostic test.

B. abortus strain RB51 is a rough rifampicin-resistant strain, which lacks the expression of the LPS O-Side Chain (OPS) [22]. Therefore, RB51 vaccination does not induce antibodies against OPS detectable by routine serological tests, which allows vaccination and test-and-slaughter policy to be performed at any age. RB51 is a naturally occurring rough mutant derived from virulent smooth strain B. abortus 2308 by serial passages on media containing subinhibitory concentrations of rifampicin or penicillin [22]. The protection against abortion and infection induced by RB51 vaccination in cattle has been demonstrated under experimental $[9,10,23,24]$ and field conditions [25-27], in herds with high and low brucellosis prevalence.

Immunization with S19 or RB51 is generally performed in young female calves in a single dose by intramuscular or subcutaneous injection. However, in zones of high prevalence of brucellosis, massive vaccination, including adult cows and even pregnant cows, is performed employing RB51 [26-28]. Vaccination of pregnant cows with S19 or RB51 is not recommended because of the risk of abortion, although the reduced abortifacient characteristics of both vaccines [29]. A Spanish study estimates the rate of abortion caused by RB51 vaccination of pregnant cows in a large cattle population as low as $0.52 \%$ of all vaccinated pregnant cows [30]. However, it will be always necessary to balance the risk of abortion following vaccination of pregnant cows with the risks of a potential brucellosis outbreak. Despite the duration of immunity induced by S19 in cattle vaccinated as calves has proven to be quite long, reaching almost the entire productive lifespan of the cows $[11,12]$, evidences suggested that there is an increase in the immune response induced by primary vaccination after RB51 revaccination [15]. Regarding RB51 calfhood vaccination, so far, there are no experiments that evaluated the duration of immunity, but it is suggested that a booster vaccination is required between 4 and 5 years of age to maintain high levels of protection induced after primary vaccination [7]. Furthermore, RB51 revaccination may still be considered as a tool for increasing the herd immunity, since not all animals are completely protected after primary immunization.

In addition, as both S19 and RB51 can cause infection in humans, protective measures including safety training of the personal involved in vaccination and the use of personal protective equipment as gloves, long sleeve coats, protection glasses, and N95 masks are recommended.

\section{Immune Response Induced by B. abortus Vaccines}

Most of our knowledge on the protective response induced by both widely used B. abortus vaccines, S19 and RB51, comes from studies using mice. In murine models, a Th1 cellular immune response with production of IFN- $\gamma$, mainly by $\mathrm{CD} 4^{+} \mathrm{T}$-cells and $\mathrm{CD} 8^{+}$specific cytotoxic cells, but not IL-4, have been demonstrated as the main response following RB51 vaccination [13,31-33]. Likewise, following S19 vaccination, mice exhibited a strong Th1 immune response with production of IL-2, TNF- $\alpha$ and IFN- $\gamma$, and high levels of antigenspecific $\mathrm{CD}^{+}$and granzyme $\mathrm{B}$-secreting $\mathrm{CD} 8^{+} \mathrm{T}$-cell responses, but not IL-4 or IL-10 secretion [34,35].

In addition to Th1, T helper type 17 (Th17) cell subset was also observed to have a protective role after oral RB51 immunization [36]. Results suggests that Th17 cells may act synergistically with Th1 cells to achieve protection after vaccination, mainly mucosal immunity, since IL-17 and IL-22 were detected after oral RB51 vaccination and nasal challenge in mice and IFN- $\gamma$ knockout mice produced higher levels of IL-17 after RB51 oral vaccination [36].

In cattle, although little information is available on the protective immune profile induced by vaccination with S19 or RB51, recent data indicate that the pattern of cells and cytokines induced after vaccination is very similar to that observed in mice [15]. After S19 or RB51 vaccination, calves showed a significant increase in expression of IFN- $\gamma$ and IL-17A, mainly by $\mathrm{CD} 4^{+} \mathrm{T}$-cells, in expression of cytotoxicity markers by $\mathrm{CD} 8^{+} \mathrm{T}$-cells, in secretion of IL-6, in expression of memory markers in $\mathrm{CD}^{+}$and $\mathrm{CD} 8^{+} \mathrm{T}$-cells, besides a blastogenic response of $\mathrm{CD}^{+}$and $\mathrm{CD}^{+} \mathrm{T}$-cells [15]. After RB51 revaccination, cattle primary vaccinated with S19 or RB51 also showed a significant increase in IFN- $\gamma$ expression, proliferation of antigen-specific $\mathrm{CD} 4^{+}$and $\mathrm{CD} 8^{+} \mathrm{T}$-cells, cytotoxic $\mathrm{CD} 8^{+} \mathrm{T}$-cells and decrease in IL-6 production. However, following RB51 revaccination S19-prime vaccinated animals showed a $\mathrm{CD}^{+} \mathrm{T}$-cell dominant immune profile whereas animals prime-vaccinated with RB51 exhibited a CD8 ${ }^{+} \mathrm{T}$-cell dominant immune profile [15]. The results of this study also suggest that RB51 revaccination promotes an increase in the immune response regardless if the primary vaccination was performed with S19 or RB51, with some of the parameters assessed being even higher in animals prime-vaccinated with RB51 compared to animals prime-vaccinated with S19. These results strengthen the argument in favor of use of RB51 revaccination in regions where brucellosis is prevalent. Although it could not directly be correlated to protection, as there was no challenge study, the cell subsets and cytokine patterns found in this study strongly suggest that these may be the mechanisms used by S19 and RB51 to induce protection in vaccinated cattle. Supporting this hypothesis, other studies in cattle also showed evidences that specific cell mediate immune components are stimulated after $\mathrm{S} 19$ or RB51 vaccination, as IFN- $\gamma$ production and increases in $\mathrm{CD}^{+} \mathrm{T}$ or $\mathrm{CD} 8^{+} \mathrm{T}$ cell-responses $[14,37,38]$.

Concerning the humoral immune response after S19 and RB51 calfhood vaccination, as well as after RB51 revaccination, cattle produced high titers of IgG1 and low titers of IgG2 [15], whereas S19 
and RB51-vaccinated mice showed opposite results and developed substantial levels of IgG2 and low titers of IgG1 [39]. The exact contribution of humoral immunity in resistance to $B$. abortus infection is not quite well established, while the response mediated by cells has been proved to be crucial to overcome the infection. Moreover, there are significant differences in the pathophysiology of $B$. abortus infection between mice and ruminants, being some mice strains considered 'resistant' to infection, as they can clear Brucella spp. from spleen more quickly than 'sensitive' BALB/c mice.

Efforts to find out the principal characteristics of the immune response triggered in cattle by the two most used and successful $B$. abortus vaccine strains are essential to try to establish an ideal vaccine. The definition of immune markers correlated with protection, by mathematical modeling or evaluation of the immune response in vaccine - challenge studies, would be very helpful in the screening B. abortus candidate vaccines. Furthermore, as several studies have shown promising results using RB51 and S19 as vaccine vectors for heterologous antigens, the detailed understanding of the immune response generated by these strains could maximize their use in the development of dual vaccines.

\section{Bovine Brucellosis Control and Eradication Programs}

Due to public health importance of brucellosis and the impairment that it causes to the livestock industry, much effort has been expended to control and eradicate the disease in cattle. Several countries have adopted control measures against bovine brucellosis in order to reduce the prevalence or eradicate the disease from domestic livestock in an effort to prevent transmission to humans and mitigate economic losses. Vaccination of female calves is the central point of any brucellosis control program and is especially useful at the disease control stage, as it leads to significant reduction in the prevalence of the disease when well performed. However, the implementation and maintenance of a vaccination program alone is not enough to eradicate brucellosis. Hence, besides vaccination, most bovine brucellosis control and eradication programs also include test-andslaughter policies, surveillance and sanitary measures.

The aim of vaccination is the reduction of susceptible individuals in the population. Therefore, the success of any vaccination program depends mainly on the effectiveness of the vaccine used and its coverage in the target population. Strain 19 and RB51 are very effective in decreasing transmission and production losses caused by brucellosis, but are less effective at preventing infection by field strains $[7,9]$. Vaccines that can effectively prevent abortion are able to reduce the economic losses caused by the disease and decrease brucellosis transmission, since abortion is the key for the dissemination of brucellosis into cattle populations.

Also, the assessment of the quality of the B. abortus vaccines used is critical to the success of brucellosis control programs, because although the cost of the vaccine is just one fraction of the total cost of a control program, its quality will affect directly and dramatically the outcomes achieved. The evaluation of the quality of live Brucella spp. vaccines is usually based on in vitro criteria, including physico-chemical and microbiological tests as purity, dissociation, determination of $\mathrm{pH}$, humidity and count of viable bacteria. Nevertheless, genetic stability has recently been proposed as an additional criterion in assessing the quality of $B$. abortus vaccines $[40,41]$. Immunogenicity test performed in mouse is another alternative to assess the quality of bovine brucellosis vaccines, however as RB51 does not have cutoff points, i.e. defined protection zones $[1,42]$, specific parameters of protection must be established.

The first vaccination program was instituted in the beginnings of the 1940s by the US brucellosis control and eradication program and used the recently developed and approved S19 vaccine [43]. The main focus of vaccination was young female calves. This approach was followed by most of countries that had established or uses a vaccination program and helped to reduce the prevalence and incidence of brucellosis to very low levels [44].

In addition of the use of vaccination of young female calves, various authors had pointed out that vaccination of adult cows in infected herds could diminish the economic losses and transmission of brucellosis, and improved the control of the disease [26,30,43,45]. However, the widely use of S19 was precluded by the induction of antibodies that could interfere with the routine diagnostic tests and the risk of abortions, when used in pregnant animals. Thus, vaccination of adult animals is currently performed using RB51, which does not induce antibodies that could interfere with the routine diagnostic tests and was shown to have a very good performance in the control of brucellosis and its losses in infected herds and regions, with a very small risk of abortion $[26,27,30]$.

The vaccination strategies approved and adopted by most countries use S19 vaccination of young calves or heifers. This measure protects female animals before they join the reproductive herd and could be infected by contact with an aborted fetus or cow. Some brucellosis control programs also use RB51 in adult cattle population, to increase vaccination coverage levels and to reduce losses in infected herds [46,47]. Three countries had modified their vaccination programs and currently just use RB51 as official vaccine: USA, Chile and Uruguay $[43,48,49]$. Those three countries have established eradication programs, since they have very low brucellosis prevalence rates. Moreover, USA and Chile have the disease restricted to some areas. Since its conditional approval in 1996, RB51 is the only brucellosis vaccine used in cattle in USA and helped the American program to reduce the number of false-positive reactors due to vaccination with S19 [43]. Similar policy was adopted by Uruguay after depicting several outbreaks in their cattle population, which was declared brucellosis-free and, in its majority, were not vaccinated with S19. Chile also adopted RB51 vaccination on those restricted areas that still had infected herds in the country [48]. In all those countries, the implementation of RB51 instead of S19 vaccination precluded the induction of anti-OPS in the vaccinated animals and permitted the adoption of other control strategies, chiefly the test-and-slaughter policy, which were fundamental to the eradication of brucellosis. Therefore, as a country or region has a decrease in prevalence and moves to eradication of brucellosis, the benefits of S19 vaccination are overcome by the problems caused by false-positive vaccinated animals. In such situations, the most logical approach to profit from the benefits of vaccination and the needed test-and-slaughter policy is the use of RB51 as the only allowed vaccine in the program.

According to the Terrestrial Animal Health Code of World 
Organisation for Animal Health (OIE) for a country to be recognized as bovine brucellosis-free it has to reach the following conditions: (i) brucellosis has to be a compulsory notifiable disease; (ii) the entire cattle population has to be under official veterinary control; (iii) the brucellosis rate of infection cannot exceed $0.2 \%$ of cattle herds; (iv) official serological surveys have to be conducted periodically in the herds; (v) no animal should be vaccinated against brucellosis in the last three years; (vi) all positive animals have to be slaughtered; and (vii) all animals entering the country have to come from an officially brucellosis-free country or region.

For a country to move towards the eradication of bovine brucellosis, the knowledge of the points the lead to the success and failure of brucellosis eradication in different countries is essential. Therefore, the main strategies employed in association with vaccination in control, eradication or epidemiological surveillance programs of countries that achieved the eradication of bovine brucellosis or are seeking this status are thereafter discussed.

The other major strategy for the control and eradication of brucellosis is the test-and-slaughter policy, which is recommended by the OIE and is commonly used [44,50,51]. Infected animals, detected by indirect or direct methods, are separated from the herd and slaughtered, thus reducing the transmission rate in the herd. The most effective programs using test-and-slaughter policies compensates the farmers for the slaughtered animals, based on market prices of individual animals, breed and type of exploration (dairy or beef) $[44,51]$.

To increase the detection rate of brucellosis-infected animals, programs introduce policies to serologically test, in a mandatory basis, (i) cows and bulls or all cattle, even young stock, in abattoirs prior to slaughter, (ii) cattle in auctions and exhibitions or (iii) for transit purposes; (iv) cows older than three years, and (v) all the herds considered at risk, and to implement mandatory milk ring test in dairy farms [44,48,50-52]. Those policies should be implemented gradually in a rational basis as far as the animal health services are prepared to enforce them.

Positive herds should be tested regularly, in a 30 - to 180 -day basis, until two or three consecutive negative tests of all animals were obtained. During this period, the herds should be put on quarantine to avoid the dissemination of the disease.

Depopulation is also a strategy that could be used in large herds or in late eradication stages of a program. It has a high cost, but it could be much faster to implement and less risky for the maintenance or dissemination of brucellosis.

In some countries, as it was observed in USA and Canada, brucellosis control programs must also be aware of brucellosis in wildlife and its possible links to the disease in cattle. If wildlife represents reservoirs of Brucella spp for cattle, specific measures must be adopted to control the disease in such situations [44,51].

Many programs also classify herds or regions according to their sanitary status in a progressive way until they obtain the brucellosisfree status $[43,49,53]$. This policy could stimulate the farmers or State agents to keep continuously moving towards the brucellosis-free status and clearly estates which is the status of a farm or region on a certain time point.
The combination of the vaccination program with many of those control measures results in a much rapid decrease in incidence and prevalence of brucellosis in cattle, leading to a much faster eradication of the disease.

\section{Final Considerations}

Vaccination with available vaccine strains, essentially S19 and RB51, even though we still do not have an ideal vaccine, remains the most successful method for the prevention and control of brucellosis in cattle, being a critical component of most brucellosis control and eradication programs throughout the world. Besides the vaccines, the experience of countries that reach the brucellosis-free status has shown that it is imperative the use of complemental control and surveillance measures with cooperative actions of the private sector and governments. The search for an ideal vaccine passes through the better understanding of how existing vaccines confer protection in the target species, cattle. Some of the answers on how the proved vaccines induce protection in cattle recently started to be available, therefore, the future directions should be seek to find the immune markers correlated with protection.

\section{Acknowledgments}

A.P. Lage was indebted to Conselho Nacional de Desenvolvimento Científico e Tecnológico - CNPq for his fellowship. EMSD thanks CNPq and Capes (Ciência Sem Fronteiras 8808-13) for her fellowship as well. APL lab was supported by CNPq, Fundação de Amparo à Pesquisa do Estado de Minas Gerais - Fapemig and FEP - MVZ.

\section{References}

1. OIE. Bovine Brucellosis. Manual of diagnostic tests and vaccines for terrestrial animals: World Organisation for Animal Health. 2009.

2. Corbel MJE. Brucellosis in humans and animals. Geneva: World Health Organization; 2006

3. Bernues A, Manrique E, Maza MT. Economic evaluation of bovine brucellosis and tuberculosis eradication programmes in a mountain area of Spain. Prev Vet Med. 1997; 30: 137-149.

4. Carvalho Neta AV, Mol JP, Xavier MN, Paixao TA, Lage AP, Santos RL. Pathogenesis of bovine brucellosis. Vet J. 2010; 184: 146-155.

5. Bishop GC, Bosman PP, Herr S. Bovine Brucellosis. In: Coetzer JAN Thomson GR, Tustin RC, editors. Infectious diseases of livestock. Texas: University Press, College Station. 1994; 1053-66.

6. Xavier MN, Paixao TA, Poester FP, Lage AP, Santos RL. Pathological, immunohistochemical and bacteriological study of tissues and milk of cows and fetuses experimentally infected with Brucella abortus. J Com Path. 2009; 140: $149-157$.

7. Olsen SC, Stoffregen WS. Essential role of vaccines in brucellosis control and eradication programs for livestock. Exp Rev Vaccines. 2005; 4: 915-928.

8. Dorneles EM, Sriranganathan N, Lage AP. Recent advances in Brucella abortus vaccines. Vet Res. 2015; 46: 76.

9. Poester FP, Goncalves VS, Paixao TA, Santos RL, Olsen SC, Schurig GG, et al. Efficacy of strain RB51 vaccine in heifers against experimental brucellosis. Vaccine. 2006; 24: 5327-5334.

10. Cheville NF, Stevens MG, Jensen AE, Tatum FM, Halling SM. Immune responses and protection against infection and abortion in cattle experimentally vaccinated with mutant strains of Brucella abortus. American J Vet Res. 1993; 54: 1591-1597.

11. Manthei CA. Summary of controlled research with strain 19. Proceedings 63rd Ann Meet US Livestock Sanitary Association. 1959; 91-97. 
12. McDiarmid A. The Degree and Duration of Immunity in Cattle Resulting from Vaccination with S. $19 \mathrm{Br}$. abortus Vaccine and its Implication in the Future Control and Eventual Eradication of Brucellosis. Vet Rec. 1957; 69: 877-879.

13. Dorneles EM, Teixeira-Carvalho A, Araújo MSS, Sriranganathan N, Lage AP. Immune response triggered by Brucella abortus following infection or vaccination. Vaccine. 2015; 33: 3659-3666.

14. Dorneles EM, Teixeira-Carvalho A, Araujo MS, Lima GK, Martins-Filho OA Sriranganathan $\mathrm{N}$, et al. T lymphocytes subsets and cytokine pattern induced by vaccination against bovine brucellosis employing $\mathrm{S} 19$ calfhood vaccination and adult RB51 revaccination. Vaccine. 2014; 32: 6034-6038.

15. Dorneles EM, Lima GK, Teixeira-Carvalho A, Araújo MS, Martins-Filho OA, Sriranganathan N, et al. Immune response induced by S19 and RB51 calfhood vaccination and RB51 revaccination in cattle. PLoS One. 2015; 10 0136696.

16. Schurig GG, Hammerberg C, Finkler BR. Monoclonal antibodies to Brucella surface antigens associated with the smooth lipopolysaccharide complex. Am J Vet Res. 1984; 45: 967-971.

17. Ivanov AV, Salmakov KM, Olsen SC, Plumb GE. A live vaccine from Brucella abortus strain 82 for control of cattle brucellosis in the Russian Federation. Animal health research reviews / Conference of Research Workers in Animal Diseases. 2011; 12: 113-121.

18. Nicoletti $P$. The effects of adult cattle vaccination with strain 19 on the incidence of brucellosis in dairy herds in Florida and Puerto Rico. Proceedings, annual meeting of the United States Animal Health Association. 1979: 75-80.

19. Koh SH, Morley FH. The effect of calfhood vaccination with strain 19 on the serological diagnosis and eradication of bovine brucellosis. Aust Vet J. 1981; 57: 551-553.

20. Mingle CK, Manthei CA, Jasmin AM. The stability of reduced virulence exhibited by Brucella abortus strain 19. J Am Vet Med Assoc. 1941; 99: 203 205.

21. Meyer ME, Nelson CJ. Persistence of Brucella abortus, strain 19 infection in immunized cattle. Proceedings, annual meeting of the United States Animal Health Association. 1969; 73: 159-165.

22. Schurig GG, Roop RM 2nd, Bagchi T, Boyle S, Buhrman D, Sriranganathan N. Biological properties of RB51; a stable rough strain of Brucella abortus. Vet Microbiol. 1991; 28: 171-188.

23. Cheville NF, Olsen SC, Jensen AE, Stevens MG, Palmer MV, Florance AM Effects of age at vaccination on efficacy of Brucella abortus strain RB51 to protect cattle against brucellosis. Am J Vet Res. 1996; 57: 1153-1156.

24. Olsen SC, Bricker B, Palmer MV, Jensen AE, Cheville NF. Responses of cattle to two dosages of Brucella abortus strain RB51: serology, clearance and efficacy. Res Vet Sci. 1999; 66: 101-105.

25. Lord VR, Schurig GG, Cherwonogrodzky JW, Marcano MJ, Melendez GE. Field study of vaccination of cattle with Brucella abortus strains RB51 and 19 under high and low disease prevalence. AmJ Vet Res. 1998; 59: 1016-1020.

26. Martins H, Garin-Bastuji B, Lima F, Flor L, Pina Fonseca A, Boinas F. Eradication of bovine brucellosis in the Azores, Portugal-Outcome of a 5-year programme (2002-2007) based on test-and-slaughter and RB51 vaccination. Prev Vet Med. 2009; 90: 80-89.

27. Dorneles EM, Santana JA, Alves TM, Pauletti RB, Mol JP, Heinemann MB, et al. Genetic stability of Brucella abortus isolates from an outbreak by multiplelocus variable-number tandem repeat analysis (MLVA16). BMC Microbiol 2014; 14: 186.

28. Herrera-Lopez E, Suarez-Guemes F, Hernandez-Andrade L, Cordova-Lopez $D$, Diaz-Aparicio E. Epidemiological study of Brucellosis in cattle, immunized with Brucella abortus RB51 vaccine in endemic zones. Vaccine. 2010; 28 : F59-63.

29. Samartino LE, Fort M, Gregoret R, Schurig GG. Use of Brucella abortus vaccine strain RB51 in pregnant cows after calfhood vaccination with strain 19 in Argentina. Prev Vet Med. 2000; 45: 193-199.

30. Sanz C, Saez JL, Alvarez J, Cortes M, Pereira G, Reyes A, et al. Mass vaccination as a complementary tool in the control of a severe outbreak of bovine brucellosis due to Brucella abortus in Extremadura, Spain. Prev Vet Med. 2010; 97: 119-125.

31. Pasquali P, Adone R, Gasbarre LC, Pistoia C, Ciuchini F. Mouse cytokine profiles associated with Brucella abortus RB51 vaccination or B. abortus 2308 infection. Infect Immun. 2001; 69: 6541-6544.

32. He Y, Vemulapalli R, Zeytun A, Schurig GG. Induction of Specific Cytotoxic Lymphocytes in Mice Vaccinated with Brucella abortus RB51. Infect Immun. 2001; 69: 5502-5508.

33. Vemulapalli R, He Y, Boyle SM, Sriranganathan N, Schurig GG. Brucella abortus strain RB51 as a vector for heterologous protein expression and induction of specific Th1 type immune responses. Infect Immun. 2000; 68: 3290-3296

34. Fu S, Xu J, Li X, Xie Y, Qiu Y, Du X, et al. Immunization of mice with recombinant protein $\mathrm{CobB}$ or AsnC confers protection against Brucella abortus infection. PloS One. 2012; 7: e29552.

35. Yu DH, Hu XD, Cai H. A combined DNA vaccine encoding BCSP31, SOD, and L7/L12 confers high protection against Brucella abortus 2308 by inducing specific CTL responses. DNA Cell Biol. 2007; 26: 435-443.

36. Clapp B, Skyberg JA, Yang X, Thornburg T, Walters N, Pascual DW. Protective live oral brucellosis vaccines stimulate Th1 and th17 cell responses. InfectImmun. 2011; 79: 4165-4174.

37. Singh R, Basera SS, Tewari K, Yadav S, Joshi S, Singh B, et al. Safety and immunogenicity of Brucella abortus strain RB51 vaccine in cross bred cattle calves in India. Indian J Exp Biol. 2012; 50: 239-242.

38. Hu XD, Yu DH, Chen ST, Li SX, Cai H. A combined DNA vaccine provides protective immunity against Mycobacterium bovis and Brucella abortus in cattle. DNA Cell Biol. 2009; 28: 191-199.

39. Vemulapalli R, He Y, Buccolo LS, Boyle SM, Sriranganathan N, Schurig GG. Complementation of Brucella abortus RB51 with a functional wboA gene results in O-antigen synthesis and enhanced vaccine efficacy but no change in rough phenotype and attenuation. Infect Immun. 2000; 68: 3927-3932.

40. Dorneles EM, de Faria AP, Pauletti RB, Santana JA, Caldeira GA, Heinemann MB, et al. Genetic stability of Brucella abortus S19 and RB51 vaccine strains by multiple locus variable number tandem repeat analysis (MLVA16). Vaccine. 2013; 31: 4856-4859.

41. Miranda KL, Poester FP, Minharro S, Dorneles EM, Stynen AP, Lage AP. Evaluation of Brucella abortus S19 vaccines commercialized in Brazil: immunogenicity, residual virulence and MLVA15 genotyping. Vaccine. 2013; 31: 3014-3018.

42. Miranda KL, Dorneles EM, Pauletti RB, Poester FP, Lage AP. Brucella abortus S19 and RB51 vaccine immunogenicity test: evaluation of three mice (BALB/c, Swiss and CD-1 ${ }^{\circledR}$ ) and two challenge strains (544 and 2308). Vaccine. 2015; 33: 507-511

43. Ragan VE. The Animal and Plant Health Inspection Service (APHIS) brucellosis eradication program in the United States. Vet Microbiol. 2002; 90: 11-18.

44. APHIS AaPHIP. National Bovine Brucellosis Surveillance Plan. USA.2012.

45. Nicoletti P. Prevalence and persistence of Brucella abortus strain 19 infections and prevalence of other biotypes in vaccinated adult dairy cattle. J Am Vet Med Assoc. 1981; 178: 143-145.

46. Luna-Martinez JE, Mejia-Teran C. Brucellosis in Mexico: current status and trends. Vet Microbiol. 2002; 90: 19-30.

47. Poester FP, Goncalves VS, Lage AP. Brucellosis in Brazil. Vet Microbiol. 2002; 90: 55-62.

48. Rivera SA, Ramírez MC, Lopetegui IP. Eradication of bovine brucellosis in the $10^{\text {th }}$ Region de Los Lagos, Chile. Vet Microbiol. 2002; 90: 45-53.

49. Aznar MN, Samartino LE, Humblet MF, Saegerman C. Bovine brucellosis in Argentina and bordering countries: update. Transbound Emerg Dis. 2014; 61: 121-133. 
50. DEFRA Departament for Enviroment, Food and Rural Affairs. Future of Brucellosis, EBL and Warble Fly surveillance. 2009.

51. CFIA Canadian Food Inspection Agency. Brucellosis. 2011
52. OIE. Brucelose bovina, ovina y caprina. Paris: OIE; 1987

53. Samartino LE. Brucellosis in Argentina. Veterinary Microbiology. 2002; 90: 71-80.
J Bacteriol Mycol - Volume 4 Issue 1 - 2017

ISSN : 2471-0172 | www.austinpublishing group.com

Lage et al. @ All rights are reserved
Citation: Dorneles EMS, Oliveria LF and Lage AP. Brucella Abortus Vaccines: Use in Control Programs and Immune Response. J Bacteriol Mycol. 2017; 4(1): 1044. 